Revue de l'Institut des langues et cultures

d'Europe, Amérique, Afrique, Asie et Australie

14 | 2011

Traduction et Ergonomie

\title{
Quelle ergonomie pour la pratique postéditrice des textes traduits?
}

Ergonomics and Postediting in Translation

\section{Louise Brunette et Sharon O'Brien}

\section{OpenEdition}

\section{Journals}

Édition électronique

URL : http://journals.openedition.org/ilcea/1081

DOI : 10.4000/ilcea.1081

ISSN : 2101-0609

\section{Éditeur}

UGA Éditions/Université Grenoble Alpes

\section{Édition imprimée}

ISBN : 978-2-84310-203-5

ISSN : $1639-6073$

Référence électronique

Louise Brunette et Sharon O'Brien, «Quelle ergonomie pour la pratique postéditrice des textes traduits ? », ILCEA [En ligne], 14 | 2011, mis en ligne le 30 juin 2011, consulté le 19 avril 2019. URL: http://journals.openedition.org/ilcea/1081 ; DOI : 10.4000/ilcea.1081

Ce document a été généré automatiquement le 19 avril 2019

(c) ILCEA 


\title{
Quelle ergonomie pour la pratique postéditrice des textes traduits?
}

\author{
Ergonomics and Postediting in Translation
}

Louise Brunette et Sharon O'Brien

1 D'après Marie-Claude L'Homme, «l'utilisation d'un logiciel de traduction automatique [TA] impose au traducteur une modulation sensible de ses méthodes de travail [et parmi] tous les outils d'aide à la traduction, le logiciel de TA est celui qui impose le plus grand nombre de contraintes» (L'Homme, 2008, p. 245). Contraintes et modulations de diverses natures couvrent par exemple la relecture de textes asyntaxiques, l'apprentissage d'un logiciel ou de plusieurs, l'acquisition des habiletés de programmation de macrocommandes et l'adaptation constante aux définitions de la qualité suivant le client ou le type de système utilisé. En matière d'ergonomie physique, il n'est même plus besoin de mentionner l'abandon définitif du papier au profit de la lecture sur deux ou même trois écrans et selon des configurations inhabituelles comme des segments de texte et la superposition verticale de ces segments.

2 Cet ensemble d'habiletés exigées depuis relativement peu de temps, en Amérique du moins, s'applique à l'activité de "postédition » $(\mathrm{PE})$, étiquette donnée à l'intervention humaine de vérification dans la chaîne de la traduction automatique. Or, la postédition comporte son lot d'exigences à propos desquelles on ne semble pas se demander pas si elles conviennent ou non aux traducteurs et aux traductrices. Par exemple, qui s'interroge sur le confort relatif du traducteur tenu de travailler simultanément avec un logiciel de TA, une mémoire de traduction, une base de terminologie et un « traducteur de dictionnaires"? Qui s'inquiète de savoir si ce mode multitâche n'est pas perçu comme une contrainte par ceux qui traduisent ? À ce jour, certainement pas les concepteurs de logiciels. Depuis 2001, à l'époque où Krings affirmait [...] «translators and MT specialists have historically had little to do with each other ", on n'a pas vu de rapprochement significatif généralisé entre les deux groupes ${ }^{1}$. 


\section{La postédition. De quoi s'agit-il ?}

3 Jusqu'au début des années 1990, et encore en 2008 avec Marie-Claude L'Homme, le terme "postédition » renvoie à une activité étroitement et exclusivement liée aux sorties des systèmes de traduction automatique. S'agissant de TA, l'environnement physique est simple et s'apparente à celui de la révision ${ }^{2}$ traditionnelle. La relectrice travaille sur deux textes : l'original et la traduction machine. D'après la littérature, matériellement, la seule différence tient souvent à ce que le texte de départ n'existe que sous forme électronique, la sortie machine prenant indifféremment la forme d'une sortie électronique ou papier (Vasconcellos, 1987), même si, en 2011, les postéditrices travaillent presque toujours sur deux écrans. Mentionnons qu'il est aussi courant d'affirmer que le travail de postédition suppose l'existence de trois textes : le TD, le TI (texte intermédiaire) et le texte traduit ou texte cible, mais nous savons que ce point de vue ne fait pas l'objet d'un consensus.

4 Ces derniers temps, toutefois, "postédition» a tendance à recouvrir plus que la vérification ou la correction des traductions produites par des logiciels de TA. Dorénavant, les textes à revoir ont une nature hybride puisqu'ils intègrent remontées de mémoires de traduction ET sorties de traduction automatique ${ }^{3}$, comme l'illustrent, entre autres, la réflexion d'Anne-Marie Robert (2010), nos recherches et notre propre expérience. De plus, il arrive qu'à l'étape dite de « prétraduction » (préparation du texte à faire traduire automatiquement), on bloque du texte qu'on ne veut pas voir traiter par la TA ou la mémoire ; le texte « intermédiaire » présenté à la relectrice comporte donc aussi parfois des éléments à traduire à partir de zéro. En d'autres termes, la postédition ne se limite pas à la correction ou à la réparation. Cette multiplicité n'est pas sans conséquence sur la pratique professionnelle. Postéditer, comme au Bureau de la traduction du gouvernement du Canada, c'est améliorer du texte, rendre du texte intelligible et, éventuellement, traduire.

5 Voilà donc définie l'activité de postédition. À la lumière des changements rapides qui touchent la traduction, peut-on supposer que cette activité va durer? Tout le laisse croire si l'on s'arrête à l'adoption massive de la TA ou de l'amalgame $\mathrm{TA}+\mathrm{MT}^{4}+$ dictionnaires dans l'industrie de la traduction (p. ex., Microsoft, CLS-Lexitech, Gouvernement du Canada). Par ailleurs, un examen rapide des colloques et congrès des deux dernières années en TA (p. ex., AMTA, EAMT) révèle clairement l'intérêt que suscite la postédition. Pour certains, cette dernière devrait même éclipser la traduction à plus ou moins brève échéance. En outre, des établissements d'enseignement annoncent, projets de cursus à l'appui, leur intention de mettre en place des cours de postédition. C'est le cas de la Dublin City University (O’Brien, 2002). D'autres, comme l'Université du Québec en Outaouais, ont intégré timidement mais effectivement la postédition à leur programme. Enfin, des ateliers pour les professionnels se tiennent déjà, comme ce fut le cas au MT Summit d'Ottawa en 2009; de la même façon, les traducteurs présents au congrès de l'Association for Machine Translation in the Americas au mois d'octobre 2010 ont eu l'occasion de profiter d'une formation ${ }^{5}$ dans le domaine.

\section{À la recherche de la nature de l'acte de postédition}

6 Si la postédition est lexicologiquement définie, a-t-on cerné pour autant ce qu'est l'acte de postéditer? Pour notre part, nous nous efforçons depuis quelque temps de décrire 
scrupuleusement l'action pour mieux la comprendre, dans le but avoué de la rentabiliser davantage. Nous ne cherchons pas tant à insister sur l'amélioration de la rapidité et de l'efficacité de l'opération qu'à nous placer dans l'optique ergonomique de la satisfaction au travail de ceux qui la pratiquent. En matière d'ergonomie, les études portant sur l'implantation de méthodes de travail sont unanimes : le succès de l'opération tient à la participation des acteurs à toutes les phases et à tous les aspects du changement. Plus tôt et davantage les protagonistes sont partie du processus, meilleures sont les chances de faire accepter les habitudes qu'on cherche à faire adopter.

7 Entrepreneurs, agences et services de traduction n'ont pas attendu la parution d'études savantes sur la postédition pour implanter celle-ci, mais l'absence d'unanimité sur ce qu'ils considèrent comme une pratique exemplaire est révélatrice. Nous savons toutefois comment le marché se positionne. Le leitmotiv de la TA et des MT étant «plus vite à meilleur prix », l'industrie dicte impitoyablement sa loi. D'ailleurs, en 2001, Koby écrivait déjà dans l'Editor's Introduction à l'ouvrage de Krings, Repairing texts: "Speed and concomitant cost-savings are the drivers in the decision to implement MT. » Nous-mêmes (O'Brien et de Almeida, 2010) l'avons reconnu ailleurs : «[...] the possibility that being an experienced translator would be one of the prerequisites for meeting one of the criteria of a good post-editor, i.e. speed [...]. » Il nous apparait que, dans les études à grande échelle publiées à ce jour, la PE est vue principalement dans cette optique de TA, c'est-à-dire comme une réponse partielle à deux grands objectifs : rapidité et économie. Les consignes accessibles en matière de PE nous donnent raison. Ainsi, trouve-t-on reprises en 2003 (Allen, 2003) les directives en usage à la Commission européenne dès 1985 (et apparemment encore appliquées) : "Don't allow yourself to hesitate too long over any particular problem [...]. Don't embark on time-consuming research. Use only rapid research aids [...]." Allen fait le commentaire suivant, soulignant la notion de rapidité : «The guidelines [...] show that a methodology of post-editing tends to be primarily based on the philosophy of dealing with turnaround time constraints. » Dans l'étude préalablement citée et menée par de Almeida et O'Brien, nous affirmions, à propos de la localisation :

Our summary of the skills required to ensure good PE performance would be: [...] 2- The ability to carry out the post-editing task with reasonable speed, so as to meet the expectations of daily productivity for this type of activity (approximately 5,000 words postedited per day, on average) [...].

8 Malgré ses prises de position et ses attentes bien nettes, et mis à part le besoin de rapidité, le marché ne semble donc pas vraiment savoir ce qu'il entend par une bonne postédition. D'ailleurs, à l'heure actuelle, on ne peut prétendre à l'existence d'une systématisation de la postédition tant est étendu le catalogue de directives la concernant. Les guides de postédition se distinguent par leurs objectifs mêmes. On ne s'en étonnera pas puisque les systèmes de TA utilisés varient considérablement selon qu'ils sont conçus sur mesure ou qu'il s'agit de systèmes commerciaux. La postédition doit donc s'adapter. À titre d'illustration, mentionnons que, dans certaines circonstances, la postédition prend en compte les faiblesses (syntaxiques, par exemple) de rendement des systèmes de TA (p. ex., Pan American Health Organization) auxquelles elle prévoit des parades, et que dans d'autres, toutes les décisions dépendent de la personne qui postédite (p.ex., MultiTrans de MultiCorpora). Certaines directives mettent l'accent sur les erreurs à corriger (Localization International Standardization Organization), d'autres sur les attitudes à privilégier (Commission européenne, voir Wagner, 1985). Cependant, certaines sont immuables : prendre la mesure de la mission et de la portée du texte; distinguer entre corrections essentielles et améliorations ; respecter le principe de destination. 
9 Une fois encore, nous reprenons à notre compte les propos de Allen au sujet des consignes de postédition :

[...] we are aware that the majority of translation/localization agencies and in-house translation departments that are conducting post-editing in production environments are creating their own sets of post-editing criteria, and in many cases are running the risk of reinventing the same wheel. (Allen, 2003, p. 298)

10 Ainsi, dans un service du gouvernement du Canada où nous avons fait de la postédition, nous avons reçu des consignes mises au point par les gestionnaires sans consultation d'aucune sorte avec des organismes faisant déjà de la postédition; le guide en cause diffère de celui de la Commission européenne à l'intention de ses sous-traitants, par exemple, ou de celui de GALE pour l'évaluation des systèmes de TA, ou encore de celui de la General Motors (Allen, 2003), qui insiste, on ne s'en étonnera pas, sur la terminologie ${ }^{6}$. Il n'a rien à voir non plus avec les directives systématisées données aux postéditeurs de la $\mathrm{PAHO}^{7}$, organisme pionnier dans la relecture systématique des sorties machines, pas plus qu'avec les directives générales de TAUS ${ }^{8}$ (2011). Nous ajouterons par ailleurs qu'il règne encore un certain flou autour de notions apparemment simples comme postédition rapide (PER), essentielle ou minimale d'une part, et postédition classique (PEC) ou maximale, d'autre part ${ }^{9}$.

11 Absence de consensus et d'uniformité sont autant d'indices de la minceur des recherches sérieuses sur la PE. Il y en a d'autres: par exemple, à ceux qui veulent savoir ce qui différencie postédition et révision, on ne sait rien dire d'autre que : $1^{\circ}$ les erreurs à corriger ne sont pas les mêmes; $2^{\circ}$ le texte résultant peut reproduire la structure formelle du TD dans le cas de la PE ; $3^{\circ}$ la PE transporte ceux qui la font dans un univers de productivité extrême étranger aux traducteurs traditionnels. Ce n'est pas notre propos, mais ce genre d'explications ne fait guère avancer la science.

\section{Les recherches en postédition}

12 La science s'intéresse effectivement de plus en plus à la postédition. C'est Hans P. Krings (2001) qui sans conteste a donné ses lettres de noblesse à la postédition comme objet de recherche universitaire après que des pionnières comme Muriel Vasconcellos (PAHO), Anne-Marie Lauffler-Laurian (CE), Emma Wagner et Jeff Allen ont attiré l'attention sur les conditions d'exercice de cette activité professionnelle. Les choses ontelles évolué depuis Krings?

Krings a analysé la postédition dans une optique psycholinguistique et s'est intéressé surtout aux résultats qualitatifs obtenus. Pour notre part, notamment du côté de l'Europe, nous avons fait porter nos travaux sur la charge cognitive imposée aux agents de postédition à la lumière, notamment, de la corrélation entre leur expérience en traduction et leur efficacité, telle que la conçoivent les demandeurs de postédition.

14 Même si nos divers protocoles, y compris l'utilisation d'enregistreurs comme Translog ne nous ont pas amenées à des conclusions incontestables, nous avons tout de même recueilli suffisamment de données pour dresser une ébauche de la formation idéale en postédition (O’Brien, 2002), qui sera appliquée au moins partiellement dans les mois qui viennent.

15 Par ailleurs, le groupe PostEd, au Québec, a adopté une optique quelque peu différente. Même si ses objectifs de base sont les mêmes - améliorer le produit fini tout en facilitant 
le travail de postédition - et, ultérieurement, participer à la mise en place de cours autonomes de postédition et tenter de concevoir une norme minimale pour la profession, il veut se concentrer davantage sur l'aide à la création d'outils. Pour arriver à faire des suggestions aux concepteurs, PostEd applique les méthodes de l'observation contextuelle. Il part du postulat suivant: les outils sont conçus indépendamment des besoins des utilisateurs. C'est ce qu'entendait aussi Krings (2001), déjà cité, lorsqu'il écrivait : «[...] Translators and MT specialists have historically had little to do with each other.» Pour rétablir ou équilibrer la situation, PostEd propose de :

- placer les personnes, plutôt que l'informatique ou la linguistique, au cœur de la recherche ;

- définir les besoins de ceux qui font de la postédition;

- dégager des idées d'outils d'aide à la postédition ;

- faire créer à partir de données objectives les premiers véritables outils d'aide à la postédition.

Comment l'observation se déroule-t-elle? Il s'agit, en fait, d'étudier les pratiques in vivo, c'est-à-dire dans un environnement non contrôlé, dans l'accomplissement d'une tâche réelle (dite naturelle) pour dégager les habitudes professionnelles de la personne chargée de postéditer et répondre ultérieurement à ses besoins en technologie. Nous travaillons avec douze sujets du secteur privé ou appartenant à des organismes internationaux. Nous les avons enregistrés dans des conditions idéales, suivant notre méthodologie, c'est-à-dire dans l'accomplissement de leur tâche en cours.

Les données ont été rassemblées par divers moyens audio, vidéo, cinétiques (enregistrement des mouvements de clavier et de souris, par exemple) et par questions contextuelles ou en rétrospective; il y a également collecte traditionnelle de documents (nous gardons des traces papier des documents postédités); les résultats sont analysés suivant une méthode mixte.

Les données chronométrées sont intégralement transcrites dans Transana (< www.transana.org>), logiciel de gestion des données: sont enregistrées les données chronologiques, de même que la transcription (écrite) intégrale des observations ou des réflexions des sujets. Grâce à ce logiciel, les chercheurs visualisent la méthode de travail de postédition et l'écran (les écrans) de chaque agent de postédition. Une liste des motsclés résumant en quelque sorte le contenu des commentaires ou des descriptions est créée par les chercheurs. Exemples de mots clés : surcorrection ; (faute de) ponctuation ; (faute de) sens ; terminologie ; expression de frustrations reliées au système ; affirmations relatives à la déontologie; pratiques professionnelles.

Ces identifiants appartiennent pour la plupart au catalogue de données suggérées par la littérature faisant foi dans l'industrie et empruntées à l'ergonomie du Web et aux méthodes d'enquête orientées utilisateur. Exemple : environnement physique; partage des valeurs des professionnels; partage des objectifs du groupe de travail. À ces catégories s'ajoute un catalogage ouvert des actions (grounded theory), c'est-à-dire toutes catégories que les chercheurs jugent bon de créer dans le domaine qui les concerne. Ainsi, l'équipe PostEd a-t-elle créé les catégories suivantes : recours au Web ; rédaction ; intérêt pour la technologie ; opinion sur les résultats de la postédition; idée sur l'amélioration de l'outil ; erreurs irritantes ; relations avec les informaticiens ; jugement sur l'interface. 


\section{Des réponses en matière d'ergonomie}

20 liste très partielle :

- La postédition n'est pas une tâche désagréable.

- La postédition n'est pas une tâche particulièrement agréable.

- La postédition n'empêche personne de retraduire un segment jugé inacceptable.

- La postédition efficace est toujours intégrée à d'autres technologies de TA, MT ou BdT (banques ou bases de terminologie).

- Il y a lieu d'améliorer le confort des agents de postédition.

- Il serait utile de connaître la source des documents à postéditer. S'agit-il :

- d'une mise à jour d'un document antérieurement traité ?

- d'un document nouveau mais comportant du texte identique à celui d'une traduction antérieure?

- d'un document de même nature ou non que le document de référence?

- En postédition, il serait utile de connaître l'origine technologique des solutions proposées. S'agit-il :

- de sorties de TA?

- de remontées d'une mémoire de traduction?

- de solutions issues d'une banque de terminologie, d'une BdT locale ? d'une BdT générale ?

À notre avis, une analyse poussée de ces données conduira à leur validation.

21 Si les analyses ne répondent donc pas à toutes les questions, elles fournissent toutefois des réponses pour ce qui touche l'ergonomie et mettent au jour diverses idées d'amélioration des outils existants. Il nous semble opportun et urgent de travailler sur les fonctionnalités de l'outil qui assureront :

- l'identification automatique générale des versions antérieures de tout texte issu des MT ;

- l'accès facile à ces versions ;

- l'identification automatique et intuitive des sources de solution;

- la création de mémoires de révision (correction automatique par domaine).

\section{Formation, recherche et professionnalisation}

\section{La mise au jour de besoins de formation}

Que ce soit par nos expériences pédagogiques ou par les réponses issues des analyses contextuelles, nous sommes convaincues de la nécessité de former des agents de postédition à l'université. Par exemple, presque toutes les personnes observées par PostEd déplorent leur manque de préparation aux tâches de PE. Elles donneraient sans doute raison à Krings $(2001$, p. 11): «[...] translators who are new at post-editing should be carefully trained and monitored in their early weeks of using the [MT] system [...]. » Allen (2003) va dans le même sens.

Pour l'instant du moins, ce sont les traducteurs qui semblent les plus aptes à assurer des postéditions de qualité. Comme nous l'avons précédemment mentionné, des universités et des associations professionnelles partout dans le monde occidental s'intéressent à la formation traditionnelle ou continue des futurs agents de postédition et répondent ainsi au vœu de Krings. Nous proposons que ces formations servent de laboratoires 
d'ergonomie et qu'on y soit à l'écoute des futurs postéditeurs plutôt que de tenter de leur imposer les points de vue des seuls concepteurs de logiciels ou autres techniciens.

Ces améliorations d'outils et la formation des personnes ressemblent encore à des vœux pieux. Comme on le constate facilement, bien qu'elle soit connue depuis au moins 25 ans (Emma Wagner publiait ses premières directives en 1985), la postédition se cherche encore. À ce jour, la très grande majorité de ceux qui s'y sont intéressés n'ont pas cherché à la professionnaliser et se sont peu souciés de ceux qui la pratiquent. Il est encore temps de renverser la vapeur.

Nous sommes obligées de reconnaître que nos propres préoccupations sont inspirées par celles du marché, puisque c'est pour ce dernier que nous préparons nos étudiants, mais nous allons en quelque sorte au-delà. L'objectif de rentabilité n'est certainement pas à rejeter en lui-même, mais nous considérons qu'il ne suffit pas à tracer la voie vers la professionnalisation de la PE. Professionnaliser la postédition est en effet l'orientation que nous nous sommes donnée dans des études menées de part et d'autre de l'Atlantique.

À n'en pas douter, c'est aux postéditrices, aux postéditeurs et à ceux qui les forment de décider ce que sera la postédition sur le plan professionnel. Entre les seules mains de l'industrie, cette pratique aura le statut de petit métier; spécialisée, elle pourrait être partagée entre paratraducteur ${ }^{10}$ et traducteur ou encore confiée à des traducteurs d'expérience (De Almeida et O'Brien, 2010).

\section{Des besoins de recherche accrus}

27 Nous soutenons que nous devons analyser davantage la postédition et construire autour d'elle un appareil théorique qui en mettra à plat les principes. Par exemple, certains travaux seraient en cours qui devraient nous renseigner sur la charge cognitive imposée aux postéditeurs. Concernant cette dernière, il faudra longtemps pour obtenir des résultats probants : par exemple, et sans doute à cause de l'ampleur de la tâche et des ressources à mettre en œuvre, on n'a pas encore décrit l'effort exigé des professionnels qui alternent, sur le même texte, entre la correction des correspondances partielles, la réparation des sorties de $\mathrm{TA}$ et la recherche terminologique tout en étant appelés très souvent à reprendre un texte de zéro. Or, l'alternance des tâches se pose comme une difficulté fondamentale de l'opération.

La vraie professionnalisation commence par la recherche, mais une recherche ancrée dans la pratique et menée en collaboration avec les différents acteurs de la postédition: industrie, universités, concepteurs. Une conséquence possible de pareille coopération serait la mise en place de pratiques exemplaires qui remplaceraient les trop nombreux recueils de consignes actuels et dont le seul phare est l'accélération-rentabilisation. Nos travaux visent certes le travail vite fait, mais bien fait.

\section{Conclusion}

Loin de constituer une nouveauté, la postédition, autrefois marginale, occupe depuis relativement peu de temps l'avant-scène des pratiques langagières ${ }^{11}$. En milieu professionnel et sous sa triple forme : traduction + réparation de la $\mathrm{TA}$ + vérification des MT, elle devrait s'imposer d'ici peu comme la pratique principale (Robert, 2010). Or, on n'en connaît pas véritablement la nature, puisque seulement quelques chercheurs 
universitaires et/ou professionnels de la traduction (Krings, O'Brien, Vasconcellos et PostEd) en ont fait un objet d'études. Jusqu'ici, on a surtout retenu ses exigences de rapidité et de rentabilité et l'obligation pour les traducteurs et les traductrices de s'adapter à ses contraintes techniques et cognitives.

Pour nous, la PE doit aussi tendre vers la qualité. Dans cette optique, nous voulons que la pratique reste essentiellement entre les mains de professionnels, de traducteurs formés en vertu de principes pratiques et théoriques sérieux informés par la meilleure ergonomie possible. Pour ce faire, nous mettons les professionnels au cœur de nos recherches de façon à prendre connaissance de leurs besoins et de suggérer méthodes et logiciels qui assureront leur confort et, partant, leur efficacité, comme postéditeurs.

31 Comme ce travail ne saurait se faire en vase clos, nous continuons de solliciter la collaboration de l'industrie de la traduction et de l'industrie logicielle dans ce que nous croyons être le meilleur intérêt de tous, communauté universitaire comprise. L'ergonomie maximale visant la qualité optimale, sans doute peut-on envisager dans la foulée de nos travaux la rédaction sinon d'une norme, du moins celle de pratiques exemplaires en postédition.

\section{BIBLIOGRAPHIE}

ALLEN Jeffrey, « Post-editing », dans Harold Somers (éd.), Computers and Translation: A translator's guide, Philadelphie, John Benjamins Publishing Co., 2003, p. 297-317.

BRUnETte Louise, DÉsIlets Alain, MELANÇon Christiane et PATENAUdE Geneviève, « How Translators Use Tools and Resources to Resolve Translation Problems: an Ethnographic Study » [en ligne], Proceedings of Beyond Translation Memories Workshop (MT Summit XII, Ottawa, 29 août 2009), 2009. Disponible sur <www.mt-archive.info/MTS-2009-Desilets-2.pdf> [consulté le 28 janvier 2011].

De AlmeIDa Giselle et O’BRIEN Sharon, Analysing Post-Editing Performance: Correlations with years of translation experience (EAMT 2010 - European Association for Machine Translation, 27-28 mai 2010, Saint-Raphaël, France), 2010.

KRINGS Hans P. et KoBy Geoffrey S. (éds), Repairing Texts: Empirical Investigations of MachineTranslation Post-Editing Processes, Kent State University Press, Kent, Ohio, 2001, 635 p.

L'Homme Marie-Claude, Initiation à la traductique, $2^{\mathrm{e}}$ édition revue et augmentée, Montréal, Linguatech, 2008, $317 \mathrm{p}$.

O'BRIEN Sharon, Teaching Post-editing: A Proposal for Course Content [en ligne], 2002. Disponible sur < http://www.mt-archive.info/EAMT-2002-OBrien.pdf> [consulté le 28 janvier 2011].

ROBERT Anne-Marie, « La post-édition : l'avenir incontournable du traducteur ? ", Interpreting the Future (actes du colloque organisé par le BDÜ, 11-13 septembre 2009, Berlin), 2009, 8 p.

VAsConCellos Muriel, «A Comparison of MT Postediting and Traditional Revision ", dans K. Kummer (éd.), Proceedings of the 28th Annual Conference of the American Translators Association, Medford, NJ. Learned Information, 1987. 
WAGNER Emma, « Post-Editing Systran - A Challenge for Commission Translator », Terminologie et Traduction, $\mathrm{n}^{\circ}$ 3, Commission des communautés européennes, Luxembourg, 1985, p. 1-7.

\section{NOTES}

1. Certains chercheurs en technologies langagières du Conseil national de recherche du Canada (CNRC) se sont depuis peu engagés dans cette voie.

2. Le terme « révision » est utilisé comme synonyme du terme « relecture » en usage en Europe francophone.

3. Pour certains spécialistes, comme Pierre Isabelle du CNRC, les mémoires de traduction sont de la TA.

4. En anglais, l'abréviation MT désigne la traduction automatique ; en français, elle renvoie aux mémoires de traduction. Cette identité graphique est souvent source de confusion en territoire bilingue.

5. Formation assurée par Mike Dellinger.

6. Les critères de GM sont ceux qui s'appliquent à toute l'industrie étatsunienne de l'automobile en vertu de la norme SAE J2450.

7. Pan American Health Organization.

8. Translation Automation User Society (l'appellation complète est utilisée très rarement).

9. Dans la pratique, cette différence n'existe pour ainsi dire plus.

10. Plusieurs établissements postsecondaires du Canada sont en train de mettre sur pied un cursus technique intitulé Services paralangagiers. Les titulaires du diplôme en cause seront des aides-traducteurs, c'est-à-dire qu'ils effectueront les tâches reliées à la traduction dont, par exemple, des recherches terminologiques, le nettoyage des mémoires de traduction et la prétraduction.

11. Il n'est pas jusqu'au mythique Google qui n'ait son interface de correction-amélioration des textes traduits automatiquement par ses soins.

\section{RÉSUMÉS}

Peu importe ce que l'étiquette postédition a désigné depuis 30 ans, cette pratique traductive n'a jamais fait l'objet d'études à caractère ergonomique. À ce jour, seule a importé l'efficacité mesurable de l'opération. On peut se risquer à dire que c'est là d'ailleurs l'histoire de toute l'informatisation de la traduction, qui s'est faite sans consultation des traducteurs, à de rares exceptions près. Dans l'optique de l'intégration des postéditeurs dans la chaîne de production des logiciels ou des interfaces de postédition, des études sont menées de part et d'autre de l'Atlantique pour tenter de mettre à plat l'acte réel de postédition. Nous voulons démontrer combien les recherches universitaires complètent les observations des praticiens et devraient mener à une description exhaustive des détails de l'opération.

Les résultats de telles recherches, auxquelles on adjoindra l'industrie, devraient conduire à dégager une théorie de même que des pratiques exemplaires de la postédition qui, à leur tour, serviront de base à l'enseignement universitaire. 
Whatever the meaning of post-editing has been over the last 30 years, it has never been looked at from an ergonomic viewpoint. Up to now those who implemented it just wanted to keep translation costs as low as possible and the delays as short as they can. As it has been the case with translation automation as a whole, managers and developers very rarely spoke to translators or post-editors while working on methodology or software.

With a view to integrating translators and post-editors in the development of best practices or computer aided post-editing, research projects are conducted in North America and Europe as well. This article illustrates how academic research in collaboration with software experts and practitioners could help map the act of post-editing. From there, it would be easier to draft best practices as well as university course content.

\section{INDEX}

Mots-clés : ergonomie, formation universitaire, postédition (PE), recherches, traduction

Keywords : ergonomics, post-editing, research, translation, university course content

\section{AUTEURS}

\section{LOUISE BRUNETTE}

Université du Québec en Outaouais, Canada

\section{SHARON O'BRIEN}

Dublin City University, Irlande 